\title{
Gradhiva
}

Revue d'anthropologie et d'histoire des arts

$15 \mid 2012$

Robots étrangement humains

\section{Esthétiques de la manipulation}

Marionnettes et automates au Japon

The aesthetics of manipulation. Puppets and automata in Japan

Zaven Paré

\section{OpenEdition}

Journals

Édition électronique

URL : http://journals.openedition.org/gradhiva/2350

DOI : 10.4000/gradhiva.2350

ISSN : 1760-849X

Éditeur

Musée du quai Branly Jacques Chirac

Édition imprimée

Date de publication : 16 mai 2012

Pagination : 120-143

ISBN : 978-2-35744-047-0

ISSN : 0764-8928

Référence électronique

Zaven Paré, «Esthétiques de la manipulation », Gradhiva [En ligne], 15 | 2012, mis en ligne le 16 mai 2015, consulté le 04 mai 2019. URL : http://journals.openedition.org/gradhiva/2350 ; DOI : 10.4000/ gradhiva.2350

(c) musée du quai Branly 


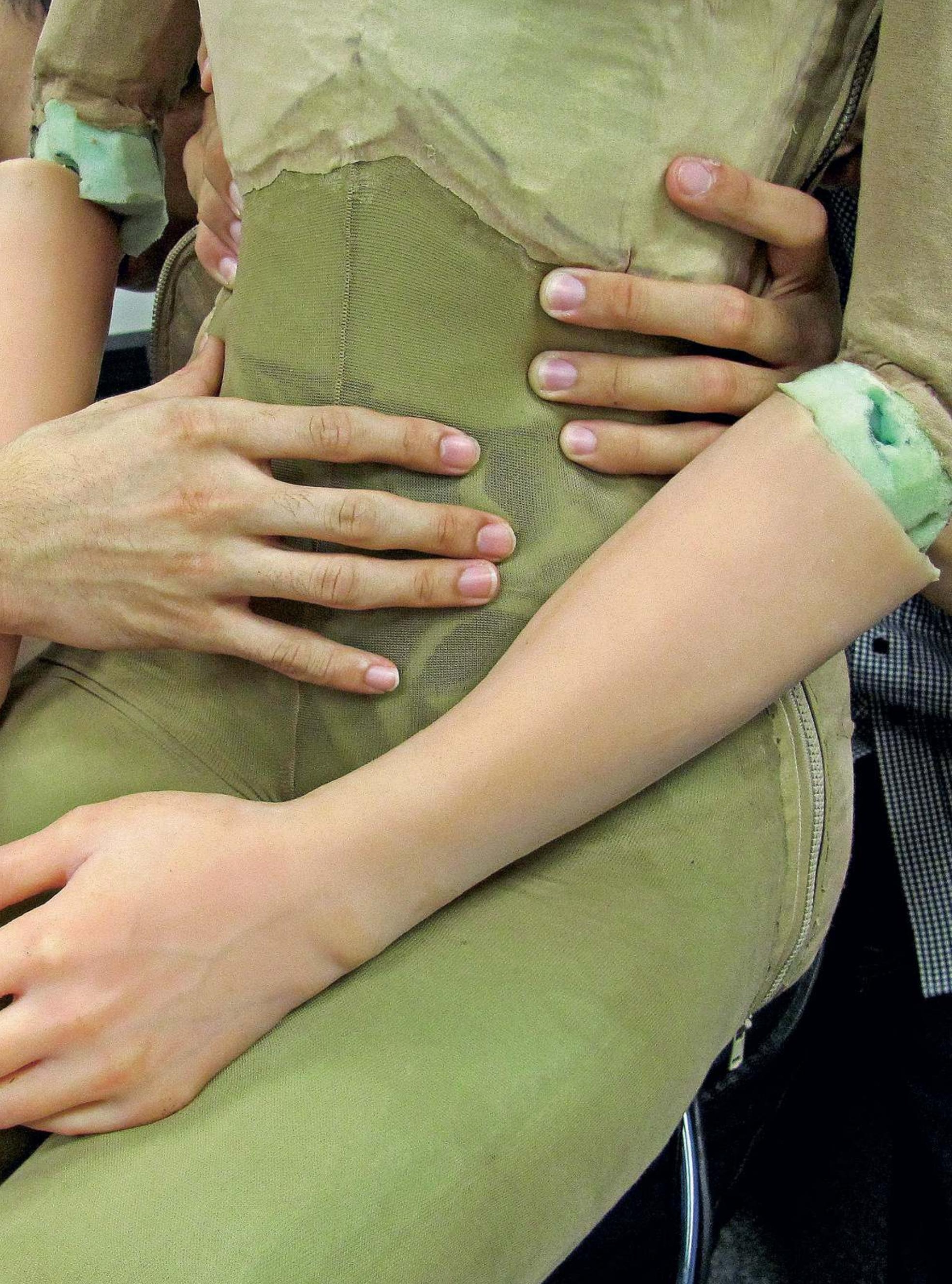




\section{Esthétiques $_{\text {de }}$ \\ la manipulation}

\section{Marionnettes et automates au Japon}

Zaven Paré

Fig. 1 Geminoid F, laboratoire ATR (Advanced Telecommunication Research International), Kyoto, photographie @ Zaven Paré.
-

1. Kleist 1998 (1801).

2. Voir l'interview du professeur Masahiro Mori dans ce même dossier et le graphique p. 28.
Le paradoxe des pantins

Le théâtre de marionnettes en général et la pantomime des poupées en particulier peuvent nous apprendre toutes sortes de choses, comme le prétendait un certain danseur C... à l'écrivain Heinrich von Kleist dans la ville de $M$, un certain hiver $1801 \ldots{ }^{1}$

Le roboticien Masahiro Mori semble se faire la même idée de certaines poupées lorsqu'il place la marionnette de bunraku sur l'un des flancs de l'uncanny valley de son graphique de 1970 : "[...] honnêtement, je ne trouve pas les marionnettes de bunraku particulièrement dérangeantes ${ }^{2}$.» Mais sous une apparence familière et sympathique, l'art de la manipulation de ces poupées nous questionne, c'est ce que je vais m'évertuer à démontrer. La singularité trouble de la robotique la plus contemporaine flirte parfois avec les mimiques des sculptures et des marionnettes les plus anciennes. Mori l'avait bien pressenti, lui qui accordait volontiers plus de vie à une sculpture du Bouddha qu'au robot le plus sophistiqué. Examinons de plus près le cas japonais. Les roboticiens japonais aiment faire remonter l'origine de leur passion aux karakuri, ces petits jouets mécaniques très en vogue $\mathrm{au}$ XVIII ${ }^{\mathrm{e}}$ siècle, qui ne sont pas non plus sans lien avec la tradition plus ancienne des marionnettes de bunraku. C'est à ces deux traditions que j'emprunterai ici mes exemples.

Dans Le Paradoxe sur le comédien, Diderot explique que le talent de l'acteur consiste à reproduire mécaniquement et sans émotion les gestes du personnage qu'il joue. L'essai de Kleist, Sur le théâtre de marionnettes, illustre aussi à sa façon cette idée : il démontre les paradoxes de la structure du corps humain par des observations sur la souplesse d'un mannequin mécanique. Une rencontre avec un danseur est le prétexte à une conversation sur le mouvement de l'acteur, et finalement à un véritable essai sur le théâtre dont Kleist analyse les tenants et 
les aboutissants. Il dépersonnalise l'interprète en parlant d'un objet, questionne le jeu en l'abordant du point de vue de la mécanique et réduit ainsi la scène à des enjeux géométriques. Si l'objet, le mouvement et le lieu n'ont pas qu'une fonction de support, ils sont cependant décrits comme les éléments participants des mécanismes d'une composition. Le danseur, en bon mécanicien, explique alors à Kleist qu'il suffit de commander des points à l'intérieur d'un pantin pour transformer leurs membres en pendules obéissant d'eux-mêmes à un centre de gravité de façon mécanique, sans que le machiniste y soit pour rien. Cette gravité détermine en partie la raison des figures, le rythme des mouvements et la grâce de la danse : "Chaque fois que le centre de gravité était déplacé en ligne droite, les membres se mettaient à décrire des courbes; souvent même, agité de manière purement fortuite, le tout adoptait une sorte de mouvement rythmique, qui ressemblait à la danse. » (Kleist 1998 [1801] : 7)

\section{La gravité dans le théâtre de bunraku}

Le théâtre de bunraku, tel qu'on le connaît actuellement, est l'une des formes japonaises de théâtre de marionnettes à s'être fixée le plus tardivement à Osaka. En 1811, le petit Jōruri d'Osaka était le seul endroit donnant régulièrement des représentations de ces spectacles traditionnels de marionnettes ${ }^{3}$, mais sous l'ère Meiji, à la fin du xix siècle, son genre connut un regain d'intérêt auprès du public4.

Si l'on regarde maintenant une marionnette de bunraku (généralement appelée ningyo) et que l'on ne s'attarde pas sur sa manipulation, on pourrait croire qu'elle incarne de manière emblématique la somme des significations de la marionnette à fils du texte de Kleist. Or il suffit de passer un peu de temps avec la troupe du théâtre national de Bunraku d'Osaka pour se rendre compte que la manipulation des marionnettes y est imprégnée de présupposés bien différents. Le bunraku est une forme d'expression artistique à part, occupant une place singulière dans le champ des créatures artificielles. Car, alors que Kleist se sert de la marionnette comme métaphore littéraire de l'impossibilité d'un retour à un " paradis verrouillé ", le ningyo, par sa savante manipulation, incarne en fait une forme condensée des contraires de la marionnette décrite par Kleist. Dans le bunraku, tout comme dans d'autres disciplines traditionnelles japonaises, le contrôle a une autre visée. Sa maîtrise n'est pas subordonnée à l'exaltation d'un retour à un état antérieur meilleur, ni à aucun paradoxe du jeu d'interprétation du comédien. C'est la marionnette qui doit sembler exprimer une émotion qu'elle ne ressent pas et non le marionnettiste, et c'est lui qui doit reproduire l'intégralité de la structure du corps humain par ses manipulations. Les manipulateurs et leurs marionnettes s'inscrivent plutôt dans la recherche d'une harmonie toujours perfectible. Comment ce principe apparemment aussi abstrait peut-il

3. Uemura Bunraken, qui en était propriétaire, le fit déplacer en 1872 à Matsushima. Son nouveau théâtre ouvrit sous le nom de Bunraku-za, donnant son nom actuel à cet art.

4. En 2003, le bunraku a été classé par l'Unesco parmi les chefs-d'œuvre du patrimoine oral et immatériel de l'humanité. s'inscrire et se lire dans la manipulation elle-même? C'est sans doute l'une des multiples virtuosités du texte de Kleist que d'arriver, en prenant pour point de départ la simple description technique d'une marionnette en fonctionnement, à des considérations d'ordre philosophique. Le même saut de la technique dans la représentation de la pensée serait facile à réaliser à partir du ningyo, mais il faudrait alors passer par l'incontournable figure du manipulateur et non rester centré sur le pantin. Chaque placé de geste est préparé, pensé et accompagné par les manipulateurs afin de gommer leur propre présence, bien qu'ils accompagnent le ningyo sur scène à la vue du public. L'adresse d'un enchaînement de 


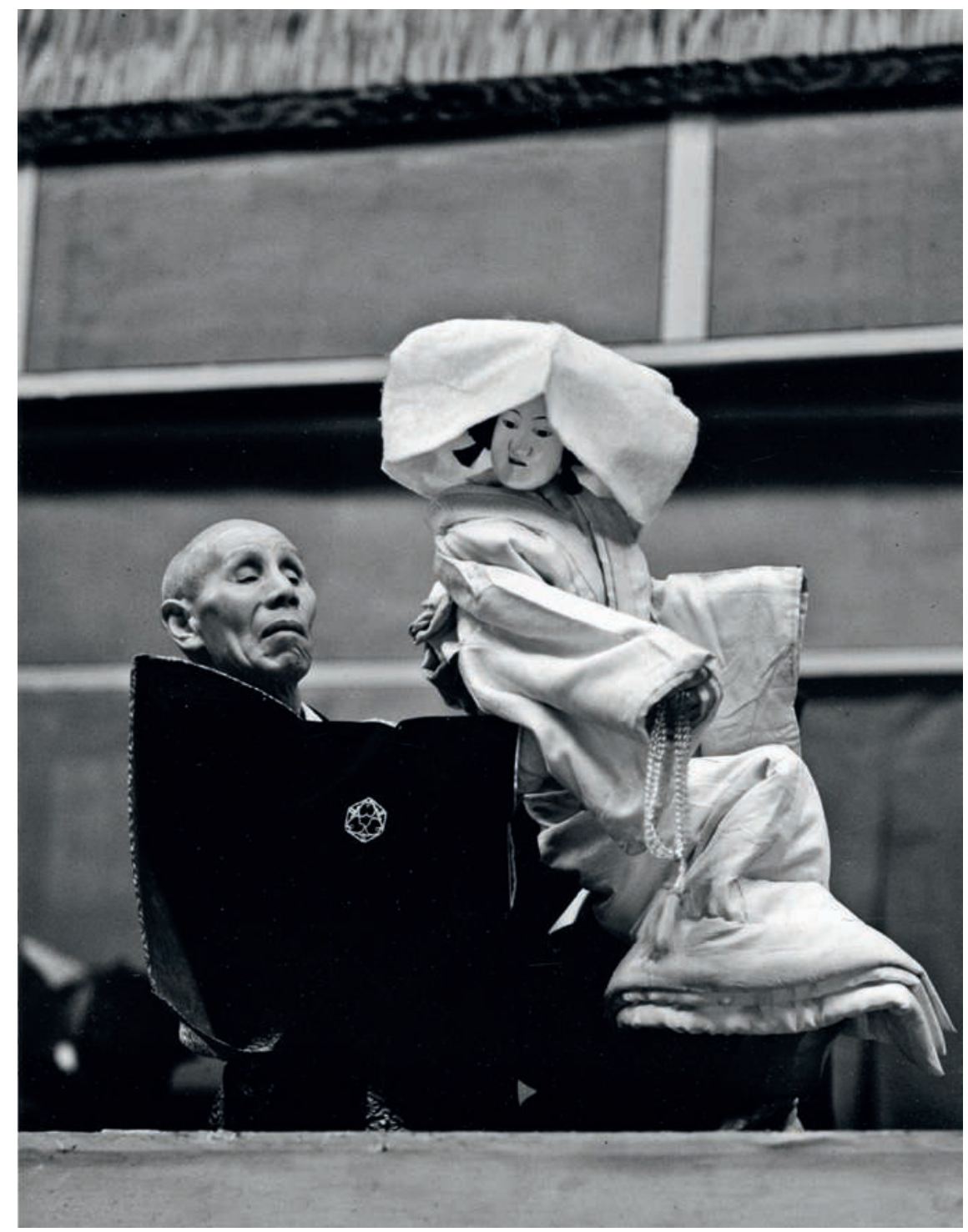

Fig. 2 Domon Ken, Représentation de "Sugawara et les secrets de la calligraphie " (Sugawara Denjyu Tenarai Kagami), 1942 @ Ken Domon Museum of Photography.

mouvements y atteint un degré de précision tel que celui-ci ne peut exister en dehors de la préparation des détails nécessaires à sa construction. Le déroulé de ce mouvement est si fluide que la composition arrive à produire l'effet contraire d'une mécanique. C'est que l'articulation mécanique y est envisagée d'emblée comme la modulation d'un flux, et c'est le manipulateur qui est à même d'opérer ce glissement de la mécanicité dans la fluidité. Le maître de bunraku peut faire oublier la pesanteur qui cloue la marionnette au sol grâce à un travail sur la légèreté et sur l'effet de présence des ningyo. Dans le bunraku, tous les mouvements, ceux des marionnettistes comme ceux des ningyo, fonctionnent alors comme une danse, dans le même rapport assez subtil des nombres à leurs logarithmes ou de l'asymptote à l'hyperbole décrit par Kleist à propos du rapport des mouvements subtils des doigts des marionnettistes et des poupées qui y sont attachées. Mais, dans les ningyo, ce rapport de l'asymptote à l'hyperbole n'est pas 
la résultante d'un mouvement pendulaire résultant des effets de la pesanteur. $\mathrm{Au}$ contraire de l'exercice de la simple gravité physique des corps des marionnettes à fils ou de certains danseurs, les mouvements d'un ningyo n'existent pas en dehors d'une sorte de mise en tension discrète, d'un calcul silencieux de la direction des gestes des marionnettistes, ni en dehors de leur déconstruction par le regard admiratif du spectateur. Par leur contrôle absolu, les marionnettistes de bunraku renforcent à chaque seconde la direction et la signification de chacun des gestes des ningyo, comme dans certaines pratiques de l'art du masque, dans le théâtre nõ par exemple. La poupée paraît alors suspendre sa chute, donnant pour ainsi dire l'impression d'arrêter son vol au-dessus de la scène du théâtre. La manipulation des ningyo se pratique en effet approximativement à un mètre du sol, derrière un panneau dissimulant notamment les jambes des manipulateurs. Les commandes des manipulations ne sont pas cachées par les frises du cintrier d'un théâtre comme dans la pratique de la marionnette à fils, mais la présence des manipulateurs est en partie tronquée par une palissade qui sépare les spectateurs de la scène. Les ningyo sont des marionnettes qui montent et semblent s'élever en puissance, et non des pantins qui chutent sous l'effet de la gravité, comme c'est le cas des marionnettes à fils.

Continuons à préciser cette différence. Chaque ningyo est normalement manipulé par trois personnes. Cette présence des trois montreurs intervient dans la qualité particulière de l'attention des spectateurs. Ainsi, le risque d'une trop grande distraction provoquée par la présence des manipulateurs est en partie compensé par l'usage d'une robe noire, le kurogo, également utilisée dans la tradition du théâtre kabuki, qui suggère l'invisibilité des montreurs cagoulés. Mais il est donné au public la possibilité de voir le maître tête nue, tout comme il pourrait contempler à loisir l'expression d'un joueur de Stradivarius qui donne vie à son instrument. S'opère alors un curieux va-et-vient entre l'observation du visage impassible du maître et le masque de la poupée. Le maître principal ou chef montreur, appelé omo zukai, manipule de sa main gauche les mouvements de la tête, nommée kashira, en tenant un bâton équipé de leviers, et de sa main droite ceux de l'avant-bras droit du ningyo. Les deux autres manipulateurs encapuchonnés de noir contrôlent respectivement l'avant-bras gauche et les jambes : le hidari zukari contrôle la main gauche de sa main droite, le ashi zukari contrôle les pieds et les jambes. Les marionnettes de femmes n'ayant pas de jambes, il doit suggérer leur présence en passant ses mains dans le bas du kimono de la marionnette. La tête et les membres sont les uniques parties rigides du corps, reliées entre elles par l'étoffe molle du kimono du ningyo. La nécessité de simuler la présence de la corporéité de la marionnette implique donc une très grande organisation et impose un haut degré de coordination entre les trois montreurs afin d'obtenir un mouvement fluide et naturel. La parfaite synchronisation des manipulations de la tête et des membres de la marionnette est nécessaire pour que le kimono ne se contente pas de tomber sous le poids de son étoffe, mais qu'il puisse au contraire donner vie ou prendre chair.

Contrairement à la marionnette à fils de Kleist ou à la description des tombés de drapés par Gaëtan Gatian de Clérambault5, par exemple, même s'il est possible de parler de plusieurs points à l'intérieur des pantins ou figures, les mouvements du corps des ningyo adoptent donc des directions qui ne peuvent être décrites par de simples lignes droites accompagnées de mouvements elliptiques. Le kimono nécessitant une technique gestuelle de pliage, ce n'est que par un jeu d'effets de manches que la pratique de ces mouvements peut en fait décrire des 


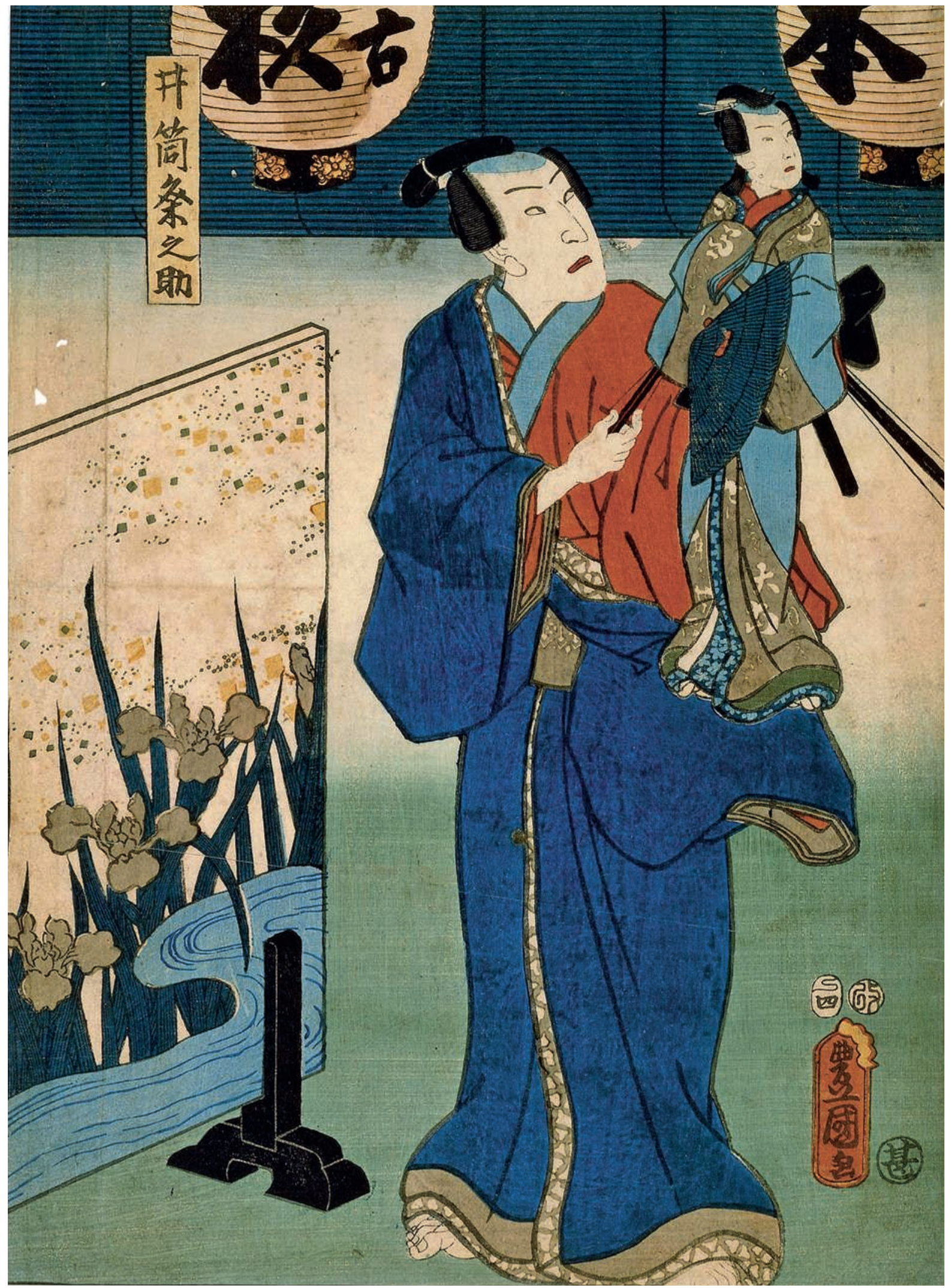

Fig. 3 Kumaseda, montreur de marionnettes japonais, s.d. Paris, Bibliothèque nationale de France. 
courbes dans l'espace. On peut illustrer ce type de figure en prenant l'exemple de l'évolution du port du kimono entre différentes générations de femmes, qui, aujourd'hui encore, l'utilisent dans divers lieux publics au Japon. Observons par exemple une jeune fille en kimono se tenant à une poignée accrochée au plafond d'un wagon dans le métro de Kyoto. On note que, la plupart du temps, sa manche descend, laissant apparaître son avant-bras ainsi dénudé. Au contraire, s'il s'agit d'une femme plus âgée, on remarquera qu'un savant geste horizontal d'ouverture de l'avant-bras avec la paume de la main vers le haut, puis de virement de celle-ci et de retour de l'avant-bras plié sur lui-même, précède la prise en main de la poignée à la verticale. Ainsi, grâce à ce mouvement de va-et-vient, d'ouverture et de fermeture dessinant discrètement un " $\mathrm{S}$ » dans le vide, la manche ne glisse pas vers le bas, le tissu est replié sur lui-même sous l'effet de son propre poids, ce qui permet de garder le bras couvert jusqu'au poignet en toute pudeur. Les manipulateurs de bunraku sont sans cesse contraints au même type de gestuelle afin de ne pas dévoiler la partie de l'avant-bras de la marionnette vêtue d'un kimono que le maître manipule à mains nues. Il n'en montre que l'extrémité, c'est-à-dire, la plupart du temps, uniquement les doigts de la poupée. Seules ces extrémités du corps du ningyo, la tête, les mains et les pieds, donnent alors ce qu'on pourrait appeler une impression de mouvement par courbes elliptiques. C'est sans doute ce rapport entre les mouvements courbes et fluides de la tête et des mains qui crée un mouvement rythmique assimilable à une danse. Les articulations des membres sont rudimentaires et requièrent une attention très précise, nécessitant une parfaite maîtrise de la part du manipulateur pour arriver à un tel effet.

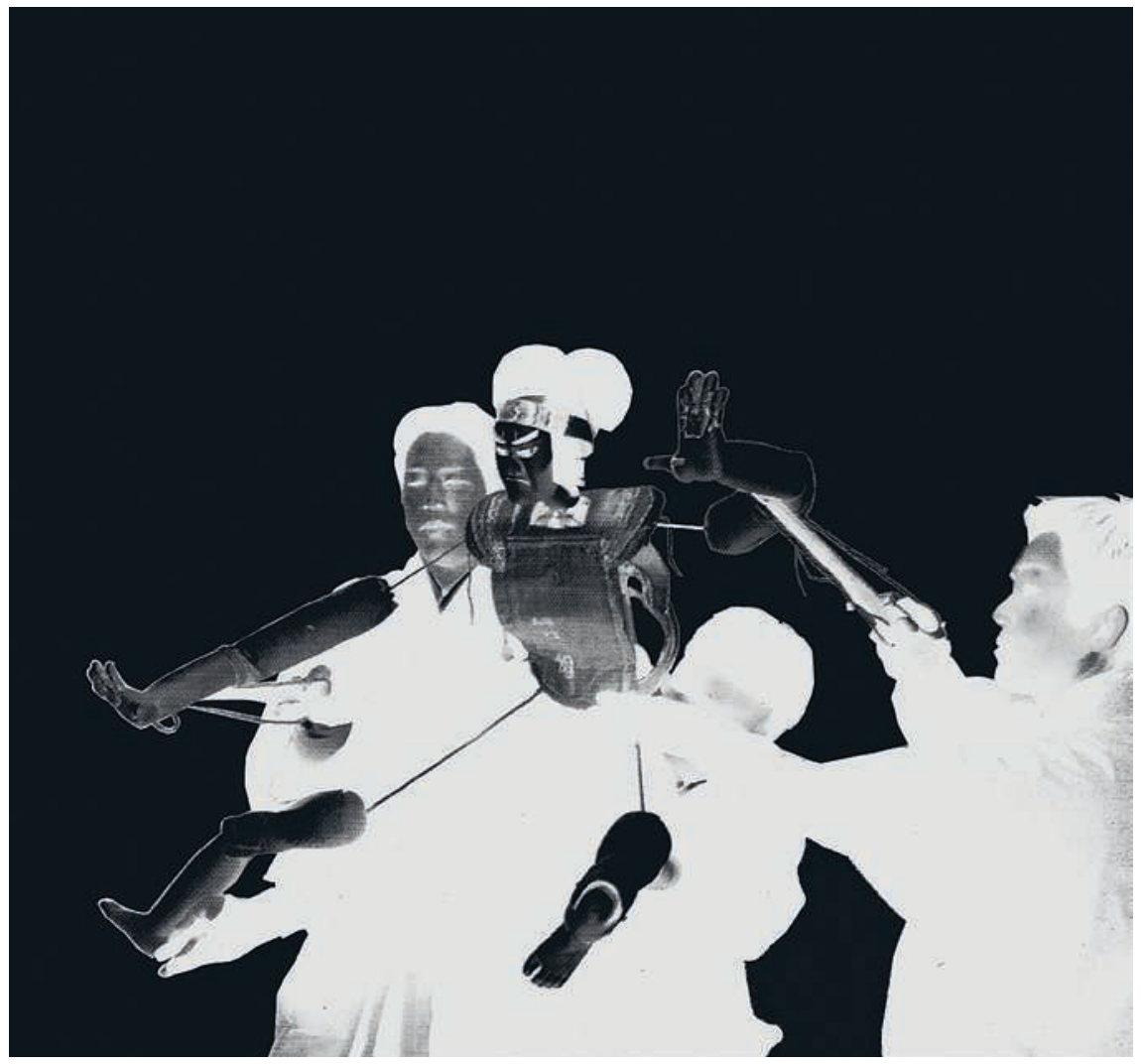

Fig. 4 Dynamique entre le ningyo et le omo zukai, le hidari zukari et le ashi zukari, in Super Premium Magazine Serai, numéro 2, p. 39, Shogakukan Publishing, Tokyo, 2009. 
Parce que chacune des parties du corps est contrôlée par un marionnettiste spécialisé, elle peut être considérée comme une marionnette. Dans un ningyo, il y a pour ainsi dire la marionnette que forment la partie droite et la tête, la marionnette de la partie gauche et la marionnette de la partie basse, constituée par les jambes. L'ensemble du corps prend appui sur celles-ci tout en étant suspendu, maintenu dans l'espace par ses montreurs. Comme la manipulation de chaque partie est l'objet d'un savoir-faire spécifique, réglé en harmonie avec le reste du corps, chacune d'entre elles répond à sa propre chorégraphie tout en se mariant à celle des autres pour former un ensemble fluide. Le maître Kanjuro Kiritake III, qui dirige la tête et la partie droite des ningyo, parle d'un flux de la partie droite et considère sa manipulation comme étant un prolongement intime et direct de son cerveau. Le ningyo est selon lui une extension naturelle de ses mains, de ses bras et de la gestuelle de son corps. Ainsi, on n'est plus en présence d'une simple chorégraphie de la marionnette et de ses parties, mais d'un véritable ballet incluant les marionnettistes, leurs gestes et leurs déplacements. Au contraire de la marionnette décrite par Kleist, le ningyo ne dépend pas d'un ou de plusieurs centres de gravité, mais de trois pôles en mouvement selon un flux de contrôles et de feedbacks complexes. Ces subtiles interactions entre les manipulateurs éclipsent, par leur virtuosité, le simple et suggestif mouvement pendulaire d'une quelconque marionnette à fils. On n'a pas affaire ici à des mouvements en ligne droite qui provoquent un balancement gravitationnel parfois fortuit, mais bien à un jeu de courbes calculées résultant d'inductions sensibles, dont le rythme compose des mouvements créés au service de l'expression harmonieuse et vitale d'un corps complet : le corps muet d'une marionnette dont on peut aller jusqu'à sentir les battements de cœur et la respiration.

\section{Les ningyo et les karakuri}

Grâce au maître de bunraku Kanjuro Kiritake III, je suis resté dans les coulisses du théâtre national de Bunraku durant un mois, puis j'ai assisté à l'enseignement de la manipulation des ningyo auprès d'un groupe de femmes du théâtre Jōruri, de la région rurale voisine au nord d'Osaka, et auprès de jeunes enfants, élèves d'un groupe scolaire voisin du théâtre d'Osaka.

Comme j'ai pu l'observer aussi dans l'art du karakuri, l'apprentissage ne passe pas ici par la transmission de connaissances orales ou écrites, mais essentiellement par l'observation du travail du maître. Le maître ne montre pas vraiment, en général il parle peut, et il démontre encore moins; en fait, il incarne la pratique. L'acquisition de la pratique de la technique, que ce soit dans la manipulation des ningyo ou dans la fabrication de karakuri, représente un défi pour l'apprenti. Celui-ci doit dans certains cas voler l'information : par exemple, surprendre le doigté de la main gauche du maître sous la robe de la marionnette lorsqu'il est chargé de la ramener dans la loge de ce dernier. Le savoir n'est pas envisagé comme un bien collectif au sens habituel du terme, étant donné qu'il repose, dans le cas du bunraku, sur des secrets de manipulation, ou encore sur des mécanismes très sophistiqués dans la conception des karakuri (où l'on n'utilise pas de plans de fabrication).

Ces singularités dans la pratique et l'apprentissage de la manipulation des ningyo permettent de comprendre pourquoi le professeur Mori a placé ces marionnettes à mi-chemin sur la courbe ascendante de son uncanny valley. 
-

6. Yoshikazu Suematsu (professeur au département d'ingénierie électromécanique de l'université de Nagoya), in Karakuri Frontier, http://suelab.nuem.nagoya-u. ac.jp/-suematsu/karafro.html.
Ce n'est pas pour rien que cette partie du graphique donne une impression de vertige : les marionnettes se retrouvent entre la partie basse du schéma, où le professeur Mori évoque les images d'un cadavre et de prothèses médicales, et son sommet, où culmine l'idéal de la représentation humaine incarné par la statuaire bouddhiste. Ce que souligne le professeur Mori en chargeant d'un tel potentiel la marionnette de bunraku, c'est que ces ningyo sont en fait des objets en devenir. Il est vrai que l'art du bunraku a cette faculté de muer l'objet immobile en présence grâce à la précision de la gestuelle de ses manipulateurs. Mais le professeur Mori ne considère pas l'activité du manipulateur à proprement parler. Il ne s'en tient, en vérité, qu'à la capacité de mouvement de la marionnette qu'il considère comme un simple objet animé, soulignant la façon dont le mouvement lui permet de devenir autre et de se rendre à nous familière par son passage de l'inerte au "vivant». Comment s'effectue ce passage? On pourrait croire que le mouvement permet essentiellement de passer d'un état à un autre, d'une expression à une autre, d'une sensation à une émotion et pourquoi pas à l'expression d'un sentiment. Certes, lorsqu'un corps est en mouvement, il passe par différents états et expressions. Il est également possible d'identifier sur ces ningyo des caractéristiques sociales, notamment par leurs attributs vestimentaires et leurs coiffures (il en existe près de quatre-vingts pour les hommes et un peu plus de la moitié pour les femmes). Mais qu'en est-il d'un visage immobile dont les traits sont peints et dont ni les yeux ni la bouche ne bougent, comme c'est souvent le cas des kashira (têtes) des marionnettes de bunraku? C'est un article de l'ingénieur en mécanique Yoshikazu Suematsu, passionné par les karakuri, qui m'a amené à comprendre ce qu'une tête de poupée japonaise peut contenir d'énoncé ${ }^{6}$. Contrairement aux visages des automates occidentaux, qui parfois expriment ou taisent leurs émotions par des mouvements d'yeux et de bouche, il est la plupart du temps impossible d'induire, de prime abord, une présence psychologique à partir des traits d'une poupée japonaise. Non pas que ces traits dissimulent quelque chose, mais le visage semble fermé sur lui-même au point d'être illisible par un observateur peu familier. À moins de développer une sensibilité à de furtifs mouvements des yeux qui se détournent de l'alignement du visage, à des clignements de paupière à peine visibles et à de fins rictus, ses potentialités expressives nous échappent largement. S'il est souvent impossible, dans ce contexte, de distinguer les traits caractéristiques d'une expression individuelle, il est probable que la lisibilité émotionnelle d'une marionnette de bunraku se décide largement ailleurs, mais où? Je ferais volontiers l'hypothèse qu'elle est justement condensée dans ce passage presque imperceptible, que les grands maîtres de bunraku aiment maîtriser, de l'immobilité à la précision d'un mouvement subtil.

Faisons un petit détour par une autre tradition japonaise, celle des automates appelés karakuri, qui est antérieure au bunraku d'Osaka. Le visage des automates y est lui aussi fixe, souvent avec les yeux mi-clos. Or il leur est curieusement prêté un grand nombre d'expressions faciales. La galerie d'exposition peu éclairée du maître Shobe Tamaya, neuvième du nom et dernier fabricant de karakuri à Nagoya, m'en a donné une brève illustration, confirmant en partie cette intuition.

L'automate japonais de "l'enfant tirant à l'arc », caractéristique du très grand savoir-faire mécanique de cet art au début du xviII siècle, est ce qu'on appelle "un automate de chambre". "Automate de table" ne serait pas tout à fait juste, puisque la plupart du temps les karakuri-ningyo étaient disposés pour être pré- 


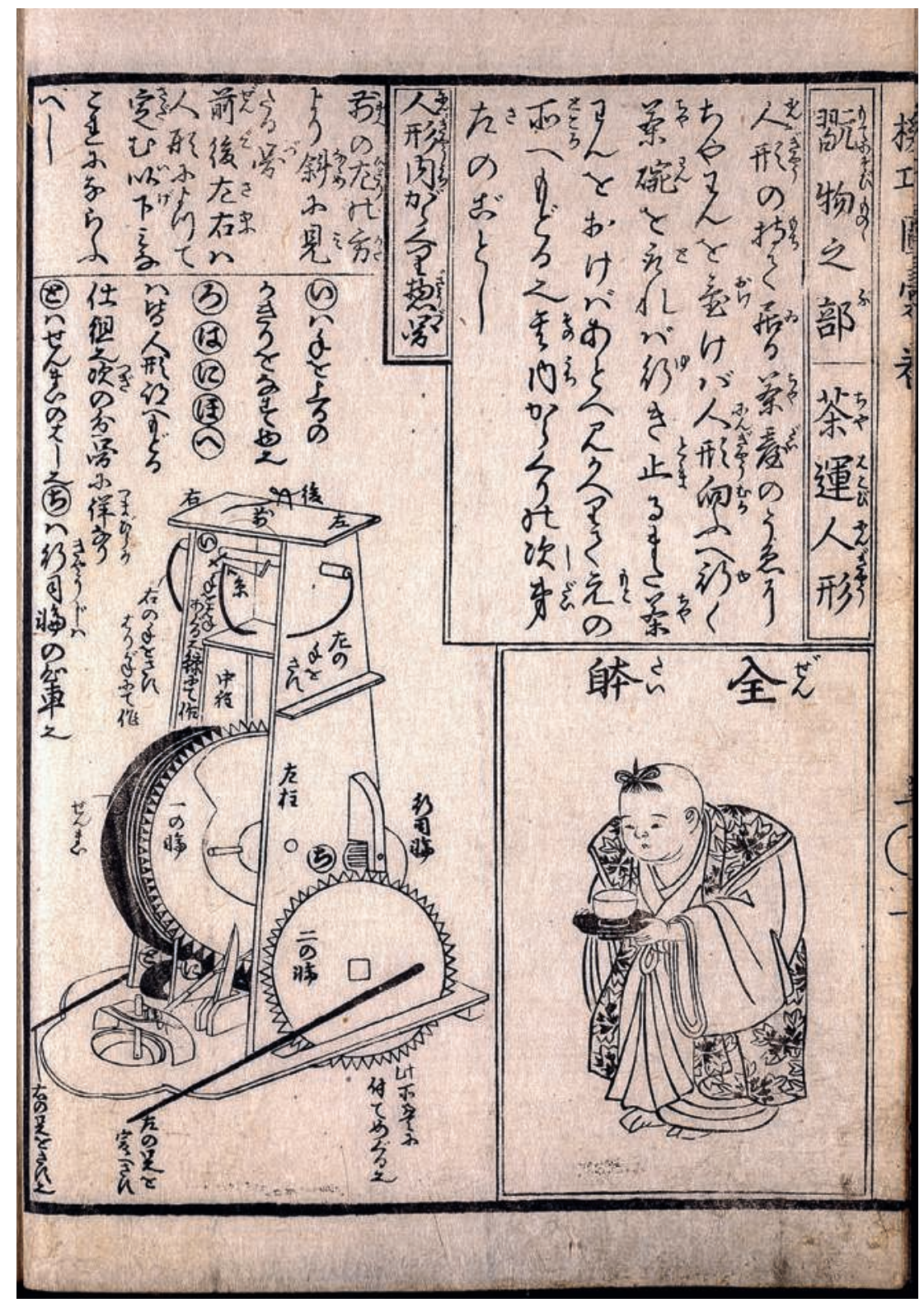

Fig. 5 Illustration du Karakuri Zui, Recueil illustré des machines astucieuses, de Hosokawa Honzô Yorinao, 1796 @ The British Museum, Londres, Dist. RMN/The Trustees of the British Museum.

sentés sur un tatami (de 182 sur $91 \mathrm{~cm}$, unité de mesure appelée jõ), ce qui déterminait la longueur de leurs mouvements, la durée de leur mécanisme et la possible complexité de leurs gestes. Il en est ainsi pour la longueur du parcours du karakuri serveur de thé et pour la portée de la flèche du tireur à l'arc. Posé sur le tatami, ce dernier prend avec sa main droite l'une des cinq flèches sur le support en éventail disposé devant lui. Comme un véritable archer, il arme son arc avec cette flèche, le bande en visant une cible à une distance d'approximativement deux mètres (un tatami) et tire. Il répète aussi minutieusement que magistralement l'opération par cinq fois, laissant estomaqué l'invité auquel est offerte la spectaculaire démonstration. L'hôte prend soin de remonter l'appareil en tirant sur une corde qui déclenche la mécanique du boîtier carré 
Fig. 6 Anonyme, acteur manipulant une marionnette, xvIII siècle. Paris, Bibliothèque nationale de France. et transparent disposé sous la figurine animée. Voici ce que décrit dans son article Suematsu, ayant assisté à l'actionnement de ce type d'automate : «Si la flèche atteint sa cible, l'archer reprend joyeusement une nouvelle flèche, mais si sa flèche suivante n'atteint pas son but, l'archer prend la prochaine flèche avec une expression qui signifie qu'il fera mieux la prochaine fois. " Rappelons qu'il s'agit d'une figurine de quelques centimètres de hauteur seulement, assise en tailleur, et dont la tête en bois ne dépasse pas $4 \mathrm{~cm}$. On est d'une part face à la miniaturisation d'une représentation et d'un mécanisme, et d'autre part devant la très grande simplicité d'expression d'un petit visage sculpté totalement impassible. Il est ainsi intéressant de constater combien le maître de karakuri est avant tout apprécié pour ses qualités de sculpteur. Il est par ailleurs étonnant de remarquer combien la charge d'expression de ce petit visage peut aller au-delà d'émotions simples telles que la joie, la peur, la rage ou la peine. Ces expressions, qui sont pourtant déjà très différenciées, semblent finalement négligeables au regard du potentiel d'intentions perçues sur le visage neutre de la poupée. Après la pause, durant laquelle le petit archer semble regarder le spectateur, sa tête s'incline vers le bas, vers son carcan; puis, alors qu'il ne fait qu'un mouvement de la tête, c'est son regard qu'il semble diriger vers la main qui tient la base de la flèche; d'un autre mouvement de la tête, il aligne une fois encore son regard en direction de la cible; la flèche est lâchée, elle poursuit inéluctablement sa trajectoire jusqu'à son but, et ainsi de suite. L'on note que le déroulement de cette action, aussi complexe qu'harmonieuse, s'exécute avec la précision et le silence d'une horlogerie raffinée. Mais, alors que l'on cherche à analyser nos propres réactions de spectateur, il est absolument impossible de dire si c'est dès la première démonstration ou après la deuxième ou la troisième que l'on se met à charger d'intentions la figure de la poupée. Il s'agit bien, avant tout, d'un divertissement, mais comment cette distraction opère-t-elle exactement? À quel moment ressent-on la joie que semble exprimer l'automate? Ou bien à quel moment encore éprouve-t-on de la déception face à l'échec ou de la résignation dans l'anticipation du prochain coup? Force est de constater qu'arrivé à la deuxième série de mouvements synchronisés de l'automate, mes impressions et mes observations n'étaient plus les mêmes. Attentif aux moindres détails, mon regard s'est sans doute alors porté davantage sur le mécanisme. Dans un premier temps, j'ai remarqué la simplicité du système de fils, remontant leviers, contrepoids, ressorts, poulies et engrenages. De prime abord, il s'agit de six types de commandes de base, mais une fois actionnées, elles révèlent une complexité extrême pour l'œil d'un simple curieux. Remarquons que le mécanisme est en grande partie en bois (jusqu'à neuf variétés), donnant l'impression d'une certaine fragilité de l'ensemble. Mais ensuite, il est fascinant de constater combien la sophistication mécanique au-dedans est transformée en mouvements harmonieux au-dehors. La découverte de cette horlogerie complexe n'empêche pas l'automate de se charger d'un supplément d'âme dès lors qu'on l'observe attentivement. Dans le clair-obscur de la pièce où le maître Tamaya effectuait sa démonstration, son visage semblait nous regarder peu à peu. Un jeu d'ombres portées sur cette tête mobile accentuait sa force suggestive, laissant entrevoir une multiplicité d'expressions. Et je ne crois pas me tromper en disant que toute personne qui entre en interaction avec un karakuri subit le même sort que moi. Car ce n'est pas l'automate qui multiplie les expressions sous nos yeux, mais c'est notre perception émotive de spectateur qui change. 


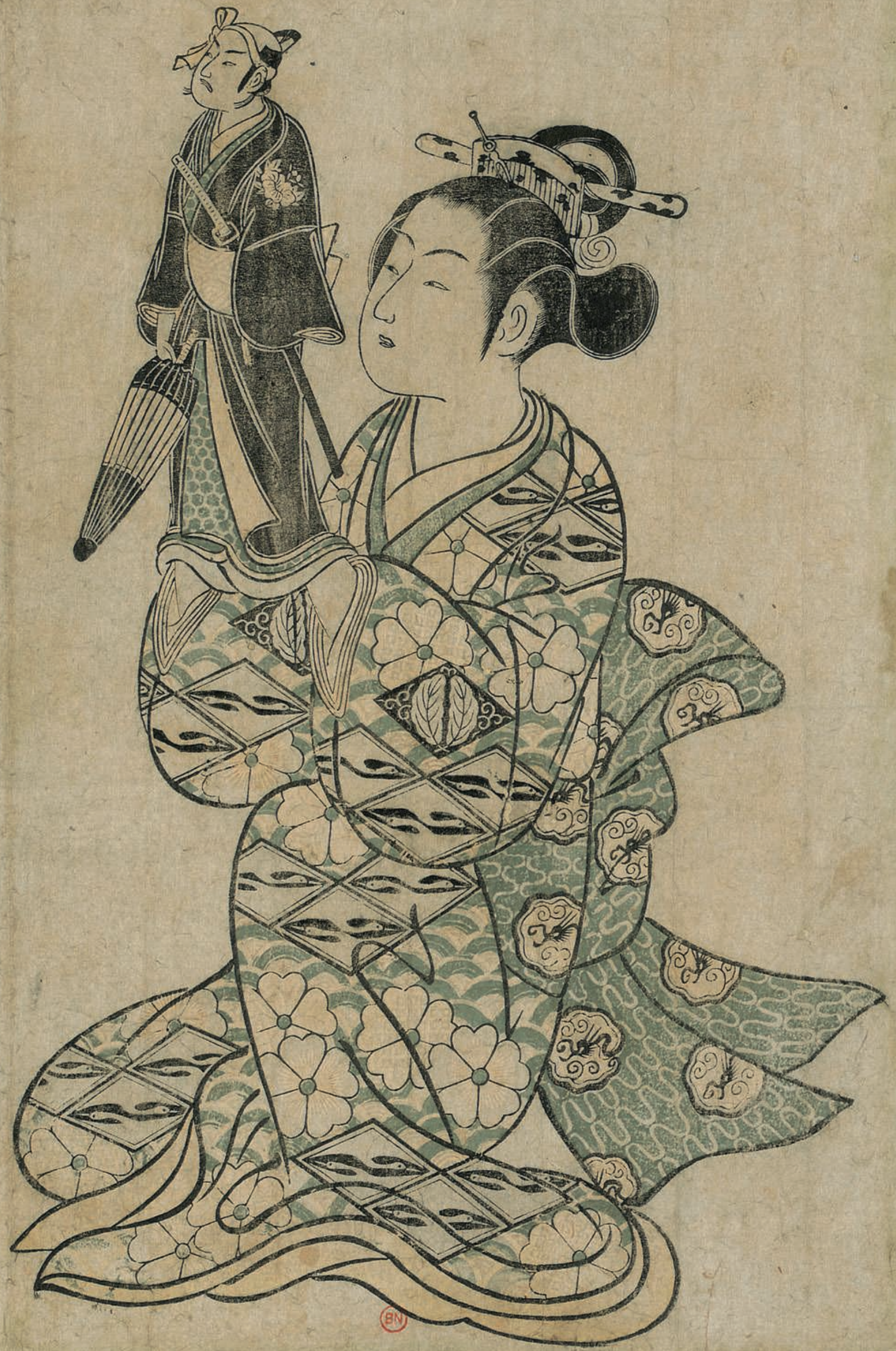




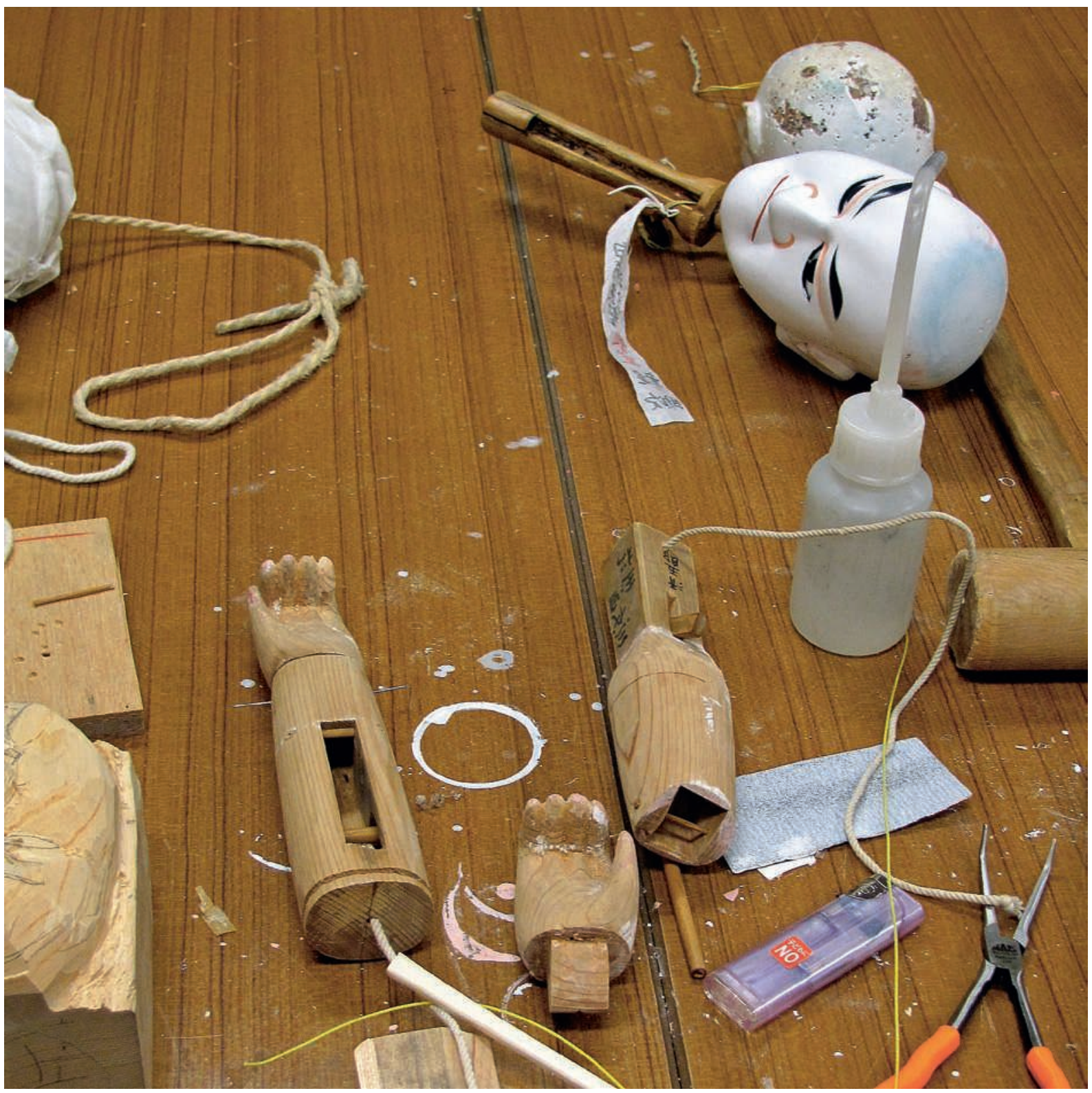

Fig. 7 Atelier de restauration et de fabrication de ningyo, théâtre du Bunraku d'Osaka, photographie @ Zaven Paré.

Par quels stades ou états psychologiques passe-t-on pour finir par éprouver de l'empathie pour cet objet mécanique? On peut distinguer dans un premier temps un rapport particulier à la dimension réduite de l'objet, puis une sorte d'empathie pour sa fragilité dans le jeu d'adresse. On finit par considérer l'archer automate comme on jugerait un véritable archer : «Pourvu qu'il ne manque pas son prochain coup! » se dit-on, tout comme on pourrait dire au fragile petit serveur de thé : «Fais attention de ne pas renverser ton bol! » En fait, qu'il s'agisse de la poupée d'un automate ou d'une marionnette, le concepteur a beau chercher dans ces objets anthropomorphiques la prouesse du jeu mécanique ou celle de la manipulation, celles-ci sont constamment remises en question par la performance devant un public. D'une part, le caractère anthropomorphique de l'objet 
est mis à l'épreuve de façon répétée lors de la représentation, ce que nous appelons performance n'étant ici que répétition mécanique, et d'autre part, il y a mise en scène d'une prise de risque mettant en jeu l'adresse, l'équilibre et la fragilité technique de l'objet. La précision de l'acte qui définit ici la prouesse technique devient la manifestation d'un trait de vérité humaine. Celui-ci réside dans le simple geste de la machine qui tient un bol en équilibre, dans l'orientation du tir à l'arc ou dans l'envol de la marionnette de bunraku. Mais essayons de décrire de manière plus détaillée les possibles interprétations des positions de la tête de l'archer dès lors qu'elle se met en action. On verra qu'il est difficile de ne pas succomber à un penchant bien connu chez les humains, qui consiste à attribuer aux choses animées un supplément d'âme.

À l'arrêt, le visage de l'automate arbore un sourire discret que l'on retrouve au coin des lèvres de certaines sculptures de la Grèce antique. C'est un sourire que l'on rencontre aussi couramment dans la statuaire bouddhiste et qui donne une impression indéniable de sérénité. La figure est aimable, enfantine et un peu joufflue, bref notre archer semble sympathique et assez bon enfant. On sourit volontiers, il nous est agréable, il semble acquiescer et avoir confiance en lui, il est même un peu fier. C'est tout juste s'il ne nous ferait pas un clin d'œil. Sa coiffure est amusante. En même temps, il apparaît digne dans son kimono, assis confortablement en tailleur. Il tient son arc à l'horizontale, comme un véritable archer, et commence son action en ramenant l'arc vers lui. À cet instant, j'ai l'impression que son regard croise le mien et j'éprouve durant une fraction de seconde une forme de connivence. Sa tête s'incline vers le bas en direction du carcan où sont disposées ses flèches. La lumière illumine son front, renforçant l'intelligence de son petit crâne, mais les traits de son visage basculent dans une légère ombre, lui conférant une expression de concentration au moment où il s'empare d'une flèche. J'imagine mal que de tels détails n'aient pas été soigneusement recherchés par le fabricant. L'automate donne vraiment l'impression de choisir la flèche qu'il va utiliser parmi les cinq disposées devant lui. Cette mise en scène de la possibilité du choix renforce sans doute notre propension à lui attribuer une intelligence. Les flèches se présentent comme un éventail de propositions. Il prend donc avec sa main droite l'une des cinq flèches, puis, alors qu'il ne fait qu'un mouvement mécanique de la tête, tout se passe comme si son regard se dirigeait vers sa main. Il pince la base de sa flèche, paraît se concentrer. L'appareil est presque silencieux et la petite poupée semble autant sourde que muette. Les plis du kimono bougent avec naturel, ce qui renforce la présence de la petite silhouette. L'automate arme son arc avec la flèche et le bande. D'un autre mouvement, sa tête s'incline sur la droite, et le creux de son œil droit bascule dans l'ombre. Il aligne avec plus de précision son regard en direction de la cible et semble plisser cet œil pour se focaliser sur le centre du cercle. Il paraît retenir son souffle; nous aussi. Je ne sais plus si je décris un véritable tireur à l'arc ou une figurine mécanique. Le temps semble soudain suspendu dans un très bref silence, rempli de la concentration qui accompagne la gestuelle fine et précise de l'archer. Ce dernier lâche sa flèche avec un sourire de satisfaction, se relâche physiquement et se détend mécaniquement. Il semble vouloir nous faire partager sa joie, son menton en avant comme en signe de défi, et l'on réalise à peine qu'il a vraiment réussi son coup. Bien sûr, la cible avait été, auparavant, soigneusement disposée pour être dans la ligne de mire du petit appareil. Cette fois, il nous regarde vraiment, très satisfait, tout en sachant rester modeste à sa manière, et surtout prêt à recommencer. 


\section{Sur le théâtre des ningyo}

Comme on a pu le remarquer dans la répétition du geste mécanique du karakuri de l'archer, la position de la tête de la poupée suggère diverses interprétations d'intentions à partir d'une succession de brefs et simples mouvements ordonnés mécaniquement. L'analyse des manipulations complexes des kashira des ningyo du bunraku peut se faire à la lumière de ces observations. En chargeant d'intentions la sculpture du visage et les mouvements qui l'accompagnent, on envisage non seulement le potentiel de la figure, mais aussi une «charge d'intention » qui varie selon les différents états psychologiques du spectateur au cours de la narration dont participe la marionnette. Lorsque le ningyo se voit attribuer ce supplément d'âme, ceci vaut jusqu'à la fin du spectacle. Tout est fait pour qu'il monte en puissance physiquement, mais aussi et surtout pour qu'il émeuve et séduise le spectateur. La manipulation gagne alors en qualité et en finesse suivant une lente progression. Le spectateur est en partie complice, et le travail des montreurs qui veulent faire croire rencontre le regard des spectateurs qui, eux, veulent bien croire. Les intentions sont donc non seulement projetées dans la sculpture du visage, mais aussi dans ses mouvements, qui répondent, sans aucun doute, à des modes de réception définis selon des codes stricts et des conventions connues et reproduites. Contrairement aux karakuri, qui n'ont que rarement des mécanismes d'yeux et de bouche, les kashira peuvent en être dotées. La relation que l'on a observée entre le regard de l'archer et sa main était circonstancielle, puisqu'elle était de l'ordre de l'action. Le regard de la kashira remplit cette même fonction, notamment dans la relation à ses gestes et à son environnement, mais, surtout, il acquiert sa charge émotive par et au cours de la manipulation. Il est possible de mesurer combien ces expressions sont renforcées dans la relation des mouvements du visage entre eux, et principalement des yeux, par la manipulation de la main droite du ningyo.

L'histoire des automates peut nous aider à mieux cerner l'originalité de la manipulation effectuée par le marionnettiste de bunraku. Prenons ici l'exemple de l'automate d'un moine franciscain fabriqué au xvi siècle par Juanelo Turriano, horloger et ingénieur de l'empereur Charles V7. Sculpté dans le bois, revêtu d'une bure, portant un chapelet et un crucifix, il est constitué d'un mécanisme de pièces de métal. D'une hauteur de trente-huit centimètres, il se déplace pareillement au karakuri serveur de thé, à ceci près qu'il trace un carré au sol comme s'il déambulait dans un cloître, un parcours certainement conçu pour que ce précieux appareil ne puisse pas tomber de la table où il était montré. Il fait des mouvements du bras, de la tête et du visage. Il se différencie des automates japonais notamment par une motricité du regard et de la bouche. Ses mouvements répétitifs et ininterrompus donnent l'impression qu'il est en état de transe. Sa tête bouge de droite à gauche, ses yeux roulent, sa bouche s'ouvre et se ferme comme si sa diction muette répétait le mime d'un Ave Maria ou d'un Mea culpa ${ }^{8}$. Mais lorsqu'il porte la croix vers son visage, non seulement ses yeux semblent se tourner vers elle, mais sa bouche parait la baiser. Ces gestes d'une très grande dévotion dans un contexte de catholicisme contreréformé sont en réalité des positions emblématiques empruntées à la prière et au sermon. Les mouvements des mains comme la position des yeux ou le type de voix nous ramènent aux conventions d'une certaine pratique oratoire : le visage doit être incliné vers la droite, de façon à ce que les yeux qui regardent le ciel se posent aussi sur les hommes; la main droite doit se tourner délicate- 


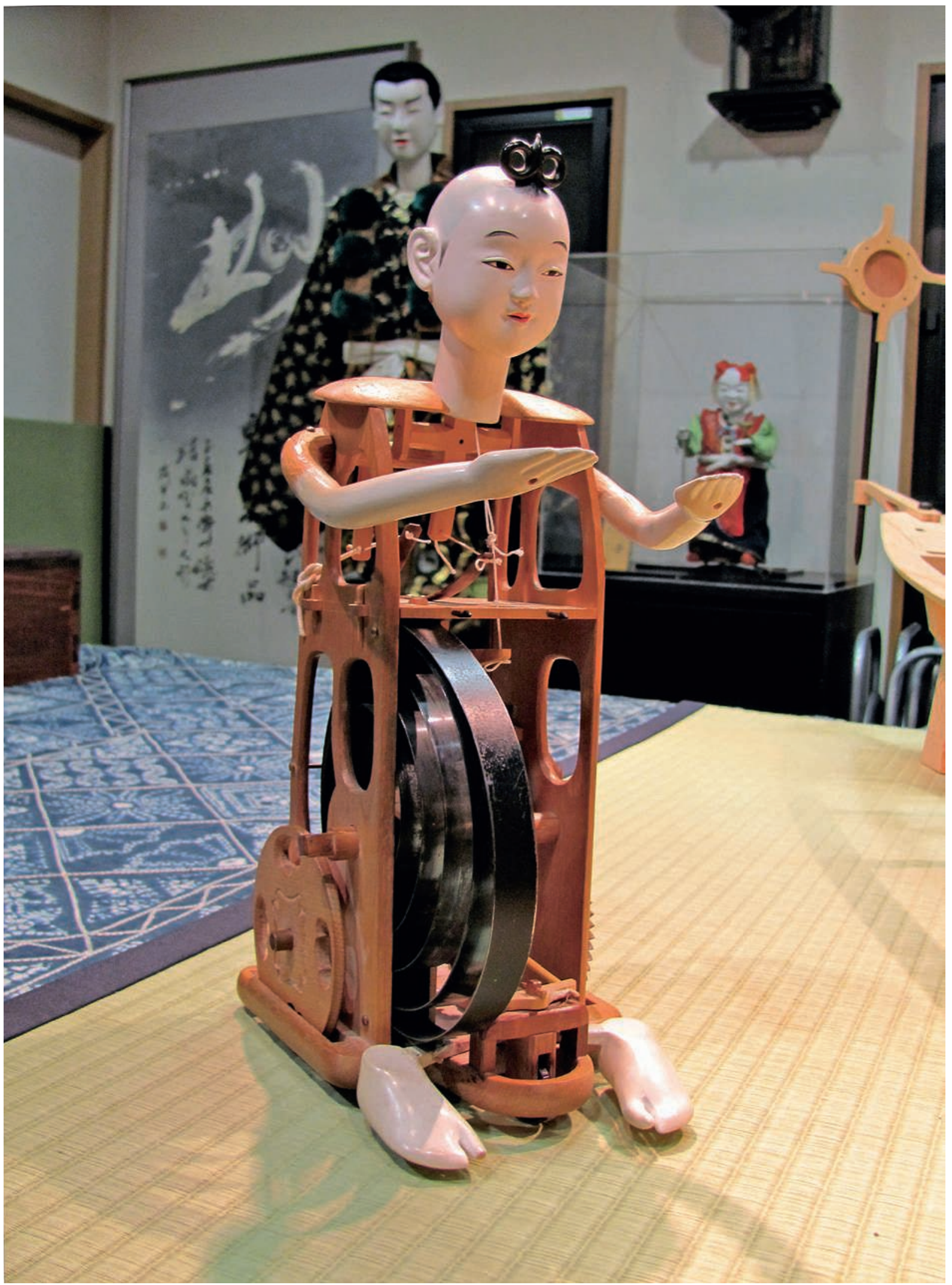

Fig. 8 Zashiki karakuri, atelier de maître Shobe Tamaya, Nagoya, photographie @ Zaven Paré. 


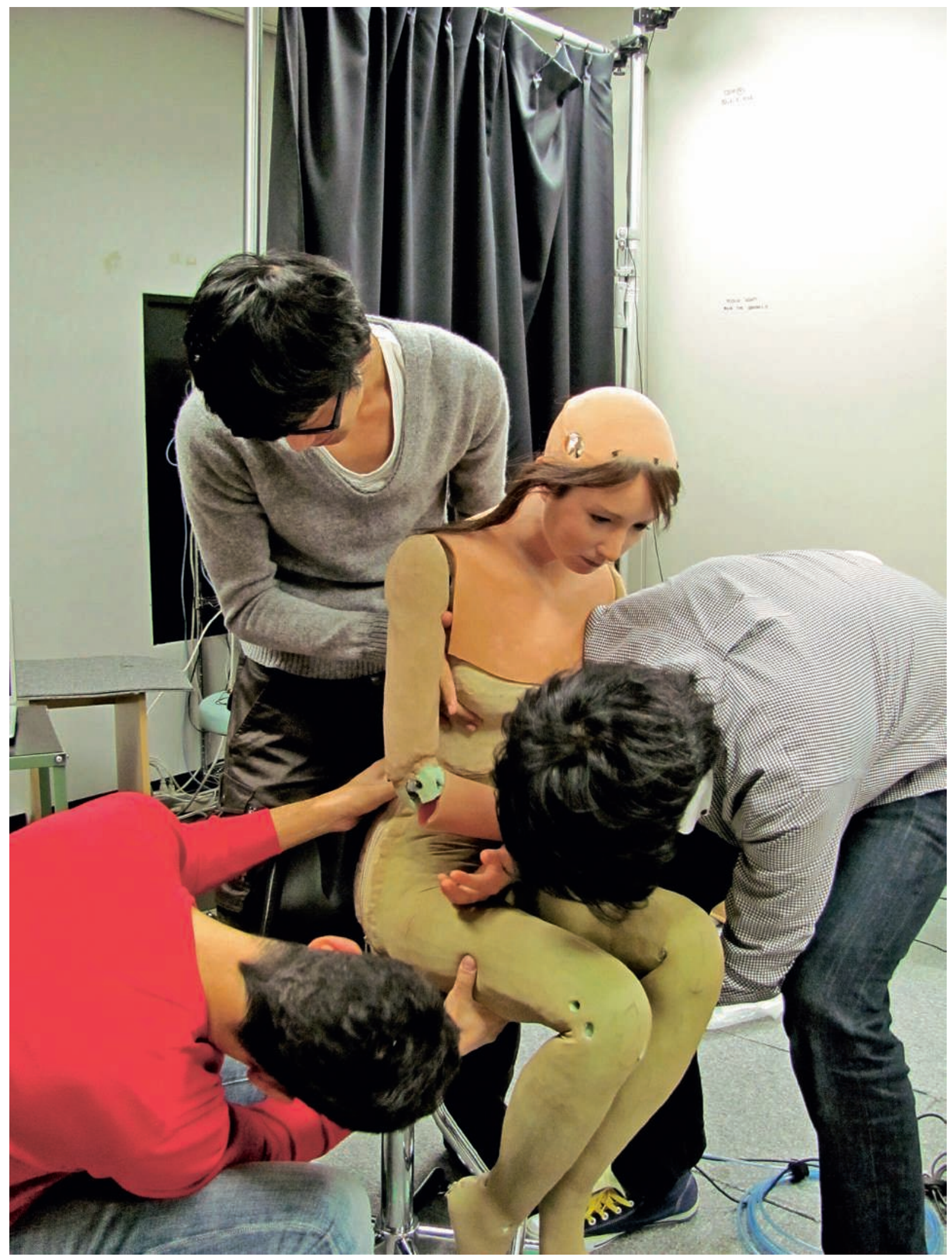

Fig. 9 Installation de Geminoid F, laboratoire ATR (Advanced Telecommunication Research International), Kyoto, photographie @ Zaven Paré 
ment vers le cœur, la gauche indiquant le ciel, sans rigidité ni tension. Dans cette description qui illustre en partie des mouvements quasi mécaniques, l'interprétation d'un geste est indissociable des pratiques et des représentations auxquelles il renvoie. Ceci est particulièrement vrai du rapport entre le regard et l'usage de la main droite en général. Il s'agit sans doute de nos deux interfaces les plus déterminantes et les plus signifiantes. Dans le cas du bunraku, il est remarquable de constater combien ces mouvements sont dans le prolongement direct des manipulations du maître, la marionnette apparaissant comme une extension physique du montreur. Il est important aussi de remarquer combien et comment ces gestes sont chargés d'expression. En évoquant le supplément d'âme qui contribue à l'effet de présence des ningyo, on a envisagé des effets difficiles à décrire autrement que par l'expérience subjective. Pour mesurer l'efficacité de tels dispositifs, il faut accepter d'en être la victime. Reconnaissons qu'il n'y a rien de plus facile. Les plus consentants d'entre nous y verront la manifestation d'une forme d'existence autre, d'un invisible qui prend soudainement une forme physique. Mon expérience des marionnettes et des automates m'amène à penser que notre propension à accorder de la vie à ce qui nous entoure ne dépend pas seulement de nous. Elle est stimulée en grande partie par la relation que la créature entretient avec sa propre représentation ou son propre corps. Certes, elle peut regarder ses pieds, mais disons qu'elle n'ira pas très loin si elle ne regarde pas devant elle. Il faut qu'elle donne l'impression d'être animée par un état de conscience, d'être à ce qu'elle fait. La coordination entre le regard et la main constitue dans la plupart de ces figures le meilleur moyen d'établir une participation, d'embrayer le spectateur dans une action en devenir. Les yeux portent un regard en synchronie ou non avec la main, le bras ou l'index qui désignent ou font montre d'ignorer. Il y a non seulement une relation, mais aussi la construction d'un espace entre ces composantes. D'ailleurs, le maître, dont le visage ne laisse transparaître aucune expression, regarde en fait dans le vide, parfois vers la main ou tout au plus dans le prolongement de la direction qu'elle indique. L'absence d'expression de son visage semble contribuer à renforcer l'idée de l'apparition d'un potentiel et virtuel effet de présence. C'est dans l'écart entre le regard et la main du ningyo que celui-ci se produit. Dans cet interstice se joue une interaction décisive. Il s'agit d'un vide où se danse un ballet aux limites du visible, qui finalement pourrait strictement se suffire à lui-même tant cette symbiose semble chargée d'intentions inconscientes, et tant elle peut résumer l'essentiel d'une pensée, se charger d'informations, de significations et d'effets de présence. Comme on a procédé avec l'archer qui projetait son regard à une distance déterminée ou avec l'automate tournant ses yeux vers le ciel, tentons de décrire ici la relation entre le regard d'un ningyo et le geste de sa main droite, animés par le même manipulateur et qui cette fois-ci montrent quelque chose au lointain.

Dans la position arrêtée, la tête des ningyo partage de nombreux points communs avec l'archer mécanique décrit précédemment. Il s'agit d'un travail de sculpture et de peinture assez similaire. La kashira est elle aussi faite d'un assemblage de deux parties creuses sculptées dans le bois, contenant une articulation ou un mécanisme. On prendra pour exemple la description du ningyo d'un personnage féminin. L'expression de son visage est semblable à celle d'un masque de nõ, outre le fait qu'ici, ses yeux peuvent bouger. Leur manipulation se fait grâce à une petite targette perpendiculaire au manche soutenant la tête et permettant d'actionner deux cordes qui contrôlent les globes oculaires par 
DOSSIER

ROBOTS ÉTRANGEMENT HUMAINS

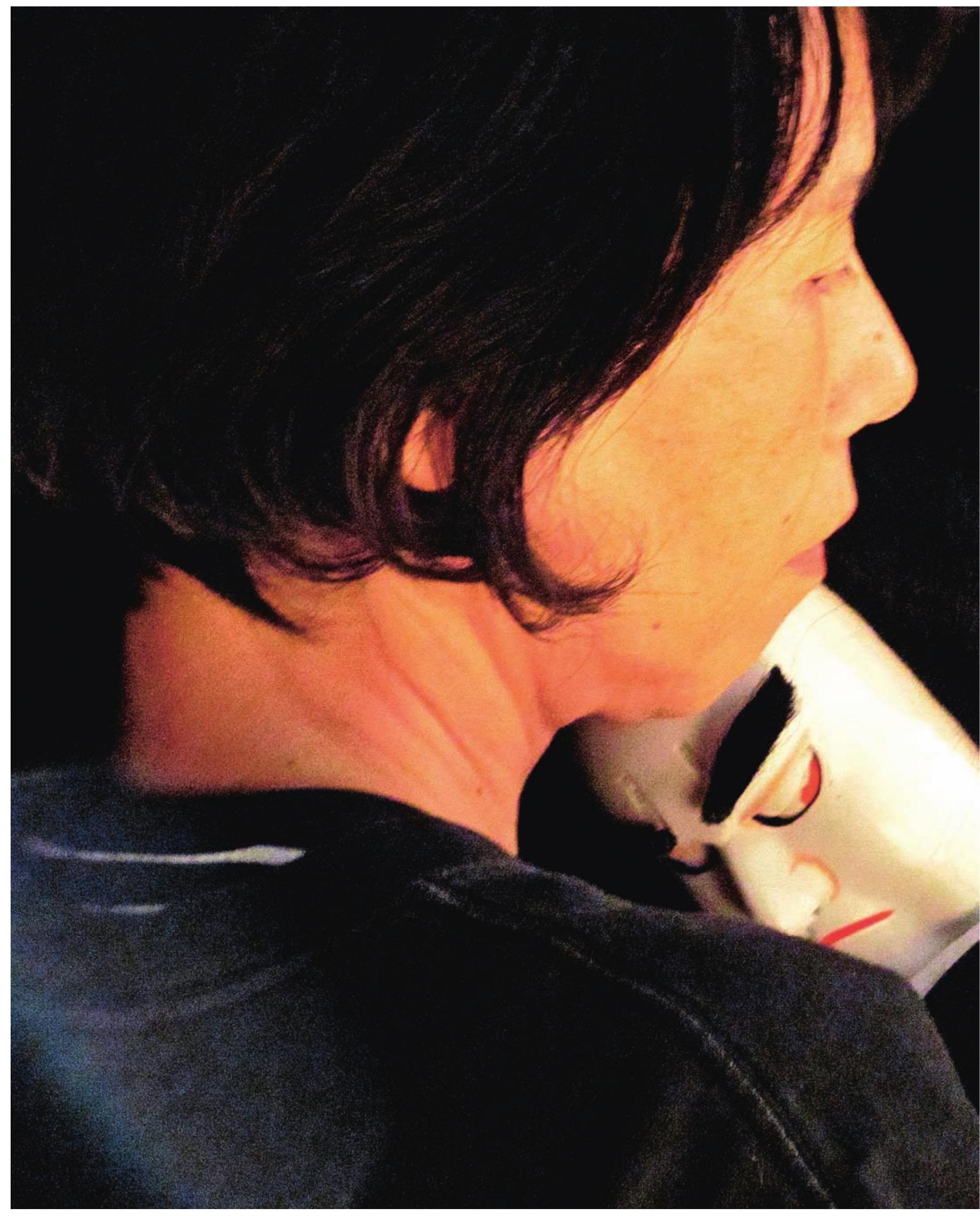



un principe de levier. Lorsque la marionnette n'est pas manipulée, son visage bascule vers l'avant sous le poids de la tête. Au repos, c'est-à-dire au point zéro de l'action, la tête est maintenue, voire retenue par le manipulateur qui tient le manche de celle-ci, sous l'articulation du cou, avec sa main gauche. Le visage laisse deviner une grande douceur qui s'exprime par son regard. Tel celui d'une geisha, au lieu d'abaisser les paupières ou de détourner les yeux, il bouge avant le regard. La tête s'incline d'abord très doucement en se penchant imperceptiblement. Les menus détails des breloques de la parure de sa coiffure semblent frissonner dans leur miroitement. La figure féminine est assise, les genoux repliés sous elle. Son kimono ornementé et chamarré ennoblit sa discrète présence. Tous les détails semblent contribuer à la situer, indiquant son rang social, sa classe d'âge, son statut familial ainsi que sa place dans la narration ou dans l'intrigue. Le conteur, ou jōruri, parle tantôt d'elle, tantôt pour elle. Comme on a pu l'observer avec le mécanisme des machines, il y a un moment de "mise en phase ". Celui-ci est introduit par le rythme des claves qui, avec l'ouverture de rideau, s'amenuise ou s'arrête de marquer le temps et définit ainsi le début de l'action, comme les douze coups de notre théâtre. Ce moment de concordance est essentiel, on ne peut pas le rater, on est là en tant que spectateur, convoqué pour ainsi dire. Le ningyo a maintenant le regard tourné vers l'horizon, par-delà le public, au lointain. La tête prend discrètement appui sur son corps. La lumière illumine le haut de son visage et sa coiffure, où brillent de vrais cheveux. Les traits de son visage oscillent doucement entre ombre et lumière. Pour aller chercher le mouvement, et ainsi indiquer l'intention de mouvement du ningyo au manipulateur de sa partie gauche, un très léger déboîtement, comme une prise d'appui quasi imperceptible de la tête, initie chaque nouveau geste. Celle-ci doit d'abord s'aligner sur son corps. Il semble alors que c'est le regard du ningyo qui guide le mouvement de sa tête. Puis, lorsqu'elle s'immobilise, le regard bouge à son tour pour induire de la vie et parfois créer une expression. Il se dirige alors vers la main maintenant alignée sur le visage. La main, en réponse au regard, bouge et s'articule d'une manière qui paraît synchronisée alors qu'elle est imperceptiblement décalée, tout en prolongeant la même intention de mouvement. La concentration induit un influx qui initie ce dernier. La gestuelle s'étend alors, fluide et déliée, sans aucune tension. L'appareil est silencieux. Les plis du kimono semblent bouger avec naturel selon les mouvements que l'on a décrits. Le personnage est bien là dans toute sa féminité, sans qu'à aucun moment il soit nécessaire d'en faire trop ou de forcer son effet de présence. Chaque mouvement paraît anticipé grâce à une pratique aiguisée. C'est l'une des prouesses du dispositif que de laisser penser qu'il n'y a pas contrôle, mais adaptation de la part du manipulateur au mouvement initié par la marionnette. Le manipulateur de la partie gauche épie le moindre frémissement, signe d'une indication, sur la nuque et l'épaule gauche du ningyo. D'un autre mouvement, la tête initie une inclinaison sur sa droite avant l'inclinaison du corps. Le corps suit la tête. Il est rarement question du contraire, à moins que ce ne soit pour effectuer un mouvement en vrille ou une danse. Telle ou telle partie du visage bascule tour à tour dans l'ombre. Le montreur tente d'aligner le regard du ningyo avec plus de précision en direction de la main et semble le faire se focaliser sur un point à une distance un peu vague au-delà de celle-ci. Il donne ainsi l'illusion d'une forme d'intériorisation, comme si le ningyo percevait par lui-même, aidé par une parfaite immobilité du reste du corps. La marionnette et son montreur semblent retenir leur souffle. Nous aussi. La main articulée 


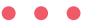

9. La pièce Sayonara a été présentée pour la première fois le 30 septembre 2010 à la triennale d'Aichi (Mini Theater de l'Aichi Arts Center). II s'agit d'un dialogue de 25 minutes entre un androïde et une actrice. fait le corps humain et les fonctions qui le reliaient à tous les autres corps, les plaçait sous la juridiction de la mécanique. En Occident, à partir de cette primauté accordée au corps, la science a abouti d'une part à l'homme-machine de Julien Offray de La Mettrie (1748) et, de l'autre, grâce à l'apport de la médecine et de la physiologie, à la formation du concept d'organisme. La structure du corps humain associée à ses réactions spontanées selon Kleist ou Spinoza prenait, dans la définition de l'homme, la place tenue jusqu'ici par les puissances spirituelles.

Alors que l'ignorance des capacités des corps était corrélative de l'ignorance des mécanismes de l'esprit, la science, la technique et l'art étaient censés délivrer l'homme de son dualisme. Or, avec l'apogée d'une société de plus en plus technique, tout repose dorénavant et indistinctement sur une certaine idée de contrôle. Au Japon, l'opposition entre le naturel et l'artificiel est amoindrie par une sorte de continuité entre l'homme et ses créations. La marionnette de bunraku, au même titre que la fonction du masque dans le théâtre nõ, en est l'allégorie. Pour le professeur Mori, les marionnettes du théâtre de bunraku sont une formidable métaphore, qu'il place sans hésitation sur la courbe ascendante de son graphique de l'uncanny valley comme s'il s'agissait de marionnettes en quête d'un idéal de vérité. En avril 2010, sur l'invitation du roboticien Hiroshi Ishiguro et du dramaturge Oriza Hirata, maître Kanjuro Kiritake III est venu au département d'ingénierie de l'université d'Osaka donner ses conseils pour la direction de l'actrice androïde Geminoid F. Geminoid F est d'une apparence ultraréaliste qui marque un grand pas dans la réalisation d'automates à apparence humaine. Elle est actuellement télérobotisée et utilisée comme plateforme de recherche en dramaturgie au sein des laboratoires, dans la perspective de remplir des fonctions d'aide à la personne. Le maître de bunraku a suggéré quelques mouvements et des postures susceptibles de donner quelque grâce au contrôle de ce nouvel androïde pour l'interprétation du personnage du robot féminin dans la pièce Sayonara9.

Dans le Japon contemporain, sans doute à l'aube d'une véritable nouvelle ère de la robotique, les ningyo qui savent danser, aimer et pleurer rappellent discrètement aux spectateurs qu'ils ont toujours la possibilité d'y croire ou non, selon leur envie. Lorsque notre regard se porte sur les robots, il s'agit en fait de la même problématique qui semble être validée ou non à chaque représentation du théâtre de bunraku. Cela se joue dans la qualité d'interprétation des poupées ou des machines et dans la représentation que nous avons de ces créatures. Comme on l'a évoqué à propos de la pratique d'un instrument de musique, la virtuosité de la manipulation des ningyo joue avec l'illusion en la produisant tout comme elle la dénonce en la montrant; mais aussi et surtout, la manipulation est la production de contrôles et la mise en scène de cet exercice.

zavenpare@gmail.com mots clés / keywords : bunraku // bunraku • karakuri // karakuri • marionnette // puppet $\cdot$ automate // automaton - Japon // Japan. 


\section{Bibliographie}

\section{Collectif}

1997 La Passion des étoffes chez un neuropsychiatre : Gaëtan Gatian de Clérambault, 1872-1934. Arles, Solin.

Courtine, Jean-Jacques et Haroche, Claudine

2007 Histoire du visage. Exprimer et taire ses émotions (du XVI ${ }^{e}$ siècle au début du XIX ${ }^{e}$ siècle). Paris, Payot.

Descartes, René

1963 (1664) Traité de l'homme. Paris, Garnier.

Diderot, Denis

1994 (1773-1777) Paradoxe sur le comédien.

Paris, Gallimard.
HosoKawa, Hanzo Yorinao

1798 Karakuri zui. Kyoto.

KANT, Emmanuel

2006 (1781-1787) Critique de la raison pure.

Paris, Flammarion.

\section{KING, Elizabeth}

2001 «Clockwork Prayer, a Sixteenth-Century

mechanical monk », in Martin L. Davies

et Marsha Meskimmon (éd.), Anthology

reconceptions: new ecologies of knowledge.

Londres, I.B. Tauris.

\section{KLEIST, Heinrich von}

1998 [1801) Sur le théâtre de marionnettes.

Paris, Mille et Une Nuits.

\author{
La Mettrie, Julien Offray de \\ 1999 (1748) L'Homme-Machine. Paris, \\ Gallimard. \\ Mori, Masahiro \\ 1970 《The uncanny valley », in Energy ? (4); \\ rééd. in The Buddha in the robot. A robot \\ engineer's thoughts on science and religion. \\ Tokyo, Kosei publishing, 1974. \\ SPINOzA, Baruch \\ 1990 (167?) Éthique. Paris, PUF.
}

\section{Résumé / Abstract}

Zaven Paré, Esthétiques de la manipulation. Marionnettes et automates au Japon - Certains robots japonais imitent singulièrement les poses des marionnettes du théâtre de bunraku. Le roboticien Masahiro Mori avait évoqué ce possible parallèle, lui qui accorde plus de vie au visage sculpté d'une marionnette ou du Bouddha qu'à un robot. Cette étude propose d'analyser successivement les manipulations des marionnettes et la mécanique des karakuri. Au Japon, l'opposition entre le naturel et l'artificiel est amoindrie par une sorte de continuité entre l'homme et ses créations. La perfection du théâtre du vivant s'y substitue à la précision des manipulations techniques des marionnettistes et des fabricants d'automates.
Zaven Paré, The aesthetics of manipulation. Puppets and automata in Japan - Certain Japanese robots imitate the postures of bunraku theatre puppets in a singular way. Roboticist Masahiro Mori, who credits more liveliness to the sculpted face of a puppet or Buddha than that of a robot, has evoked this possible parallel. This study proposes to analyse successively the manipulation of puppets and the mechanisms of karakuri. In Japan, a form of continuity between man and his creations reduces the opposition between nature and artifice. As a result, the technical preciseness of puppeteers and the virtuosity of automata manufacturers give way to the perfection of live performance. 